\title{
ANÁLISE DA INFLUÊNCIA DO TEMPO E TEMPERATURA NO PROCESSO DE RECRISTALIZAÇÃO E AMOLECIMENTO DA LIGA C272000*
}

Sidmar Aparecido Ceresani ${ }^{1}$ Givanildo Alves dos Santos Francisco Yastami Nakamoto Eduardo Guy Perpétuo Bock

\section{Resumo}

O presente estudo tem como objetivo demonstrar a cinética de recristalização e o amolecimento da liga C272000 processada na laminação a frio com uma deformação de $75 \%$. Para determinar o modelo cinético Johnson, Mehl e Kolmogorov (JMAK) foi adotado a temperatura de $450^{\circ}$ entre $5 \mathrm{~min}$. a $50 \mathrm{~min}$. Restaurando totalmente a microestrutura deformada plasticamente sendo progressiva a elevação do tempo e da temperatura. A caracterização microestrutural das amostras foi utilizado microscopia óptica e metalografia. Conclui-se que o modelo JMAK satisfaz a forma de nucleação da recristalização da liga $\mathrm{C} 272000$.

Palavras-chave: Liga de cobre; Tratamento térmico; Recristalização; Amolecimento

\section{ANALYSIS TIME AND TEMPERATURE INFLUENCE IN PROCESS OF RECLINATION AND GROUNDING LEATHER UNS C272000}

\section{Abstract}

The present study aims to demonstrate the kinetics of recrystallization and softening of the C272000 alloy processed in the cold rolling with a deformation of $75 \%$. To determine the kinetic model Johnson, Mehl and Kolmogorov (JMAK) was adopted the temperature of $450^{\circ}$ between $5 \mathrm{~min}$. To $50 \mathrm{~min}$. Totally or partially restoring the plastically deformed microstructure with increasing time and temperature being progressive. Microstructural characterization of the samples was performed using optical microscopy, metallography. It is concluded that the JMKA model satisfies the nucleation form of the recrystallization of the C272000 alloy.

Keywords: Copper alloy; Heat treatment; Recrystallization; Softening.

\footnotetext{
${ }^{1}$ Paranapanema.
} 


\section{INTRODUÇÃO}

O estado de encruamento e o resultado do processo de deformação mecânica tanto a quente quanto a frio. O resultado mais notado nesse processo e o aumento da dureza do material[1]. De acordo com a literatura, o mecanismo de migração de subcontornos aparentemente pode ser associado a altas deformações e a distribuições largas de tamanho de subgrão e a metais de baixa energia de defeitos de empilhamento (EDE). A ocorrência da recristalização está em função da quantidade de energia armazenada durante os processos de deformação plástica dessa forma, a condição microestrutural no estado de encruamento ira determinar o desenvolvimento, do crescimento e na orientação dos núcleos que originarão os grãos durante a recristalização. Para que a restauração seja total ou parcial a microestrutura inicial e os fenômenos associados ao processo de recuperação estarão relacionados ao tipo de material e a sua pureza, a deformação aplicada ao material, as temperaturas durante as etapas de recozimento e a sua deformação plástica. $O$ processo de recristalização pode ser entendido como o resultado de um conjunto de alterações na microestrutura do material em decorrência da conformação frio armazenando energia, assim quanto mais energia armazenada menos energia térmica para os átomos se rearranjarem no interior do grão, consequentemente a recristalização pode ocorrer sob determinadas condições em temperaturas mais baixas. O modelo de análise mais utilizado no crescimento de núcleos e a teoria JMAK (Johnson-Mehl-Avrami-Kolmogorov), esta equação é muito utilizado na análise da cinética global do processo de cristalização de sólidos amorfos descrevendo a dependência temporal da fração volumétrica cristalizada.

$$
Y=1-\exp (-k \operatorname{tn})
$$

Considerações do modelo matemático JMAK:

- Taxa de nucleação de novos grãos é constante.

- Taxa de crescimento dos novos grãos é linear.

- Os novos grãos são esféricos e o crescimento e isotrópico.

- A nucleação ocorre de forma aleatória e homogênea.

A recristalização e normalmente descrita por uma curva sigmoidal relacionada a fração recristalizada $\left(Y_{v}\right)$ em função do tempo $(t)$.

\section{MATERIAIS E MÉTODOS}

Neste Trabalho, foram utilizadas chapas laminadas de Latão C272000 o material foi fornecido pela empresa Paranapanema com a composição química da chapa tabela1.

Tabela 1. Composição química (\%)

\begin{tabular}{||c||c||c||c||}
\hline \hline Cobre \% & Chumbo \% & Ferro \% & Zinco \% \\
\hline \hline 63 & 0,05 & 0,04 & 36,91 \\
\hline
\end{tabular}

A chapa foi deformada na laminação a frio com uma deformação de $75 \%$ na espessura final de $0,52 \mathrm{~mm}$ a chapa foi cortada em tiras na transversal $50 \times 500$ divididas em partes 50x50 limpas e desengraxadas. Logo após, foi realizado um aquecimento em um forno tipo Mufla a $450^{\circ} \mathrm{C}$ tratando termicamente por 5 min a 50 min. As amostras foram preparadas conforme norma ASTM E-112 para o ensaio de dureza. 


\section{RESULTADOS E DISCUSSÃO}

A figura 1 mostra a evolução da microestrutura após o recozimento nota-se que o processo de recristalização ocorre plenamente somente após 25 min.
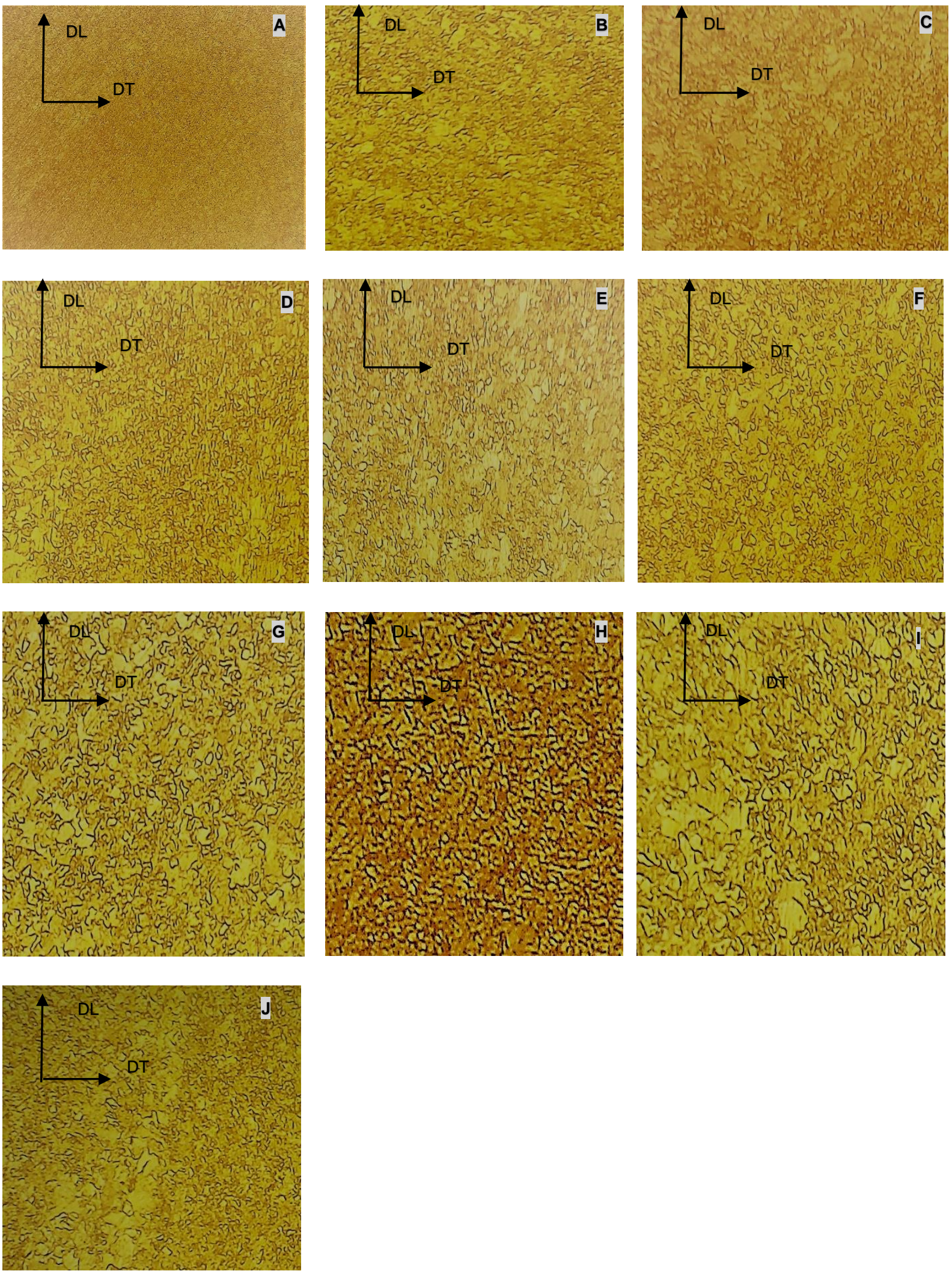
min; (e) $25 \mathrm{~min}$; (f) $30 \mathrm{~min}$; (g) 35min; (h) $40 \mathrm{~min}$; (i) $45 \mathrm{~min}$; (j) $50 \mathrm{~min}$

Este comportamento pode ser considerado como esperado para o processamento termodinâmico proposto nesta investigação à medida que a recristalização ocorre, o efeito da recuperação diminui. Observando os dados experimentais na figura2 a 0 serem comparados os tamanhos dos grãos após o recozimento no tempo de 5 min e após 30 min o crescimento do grão fica estável.

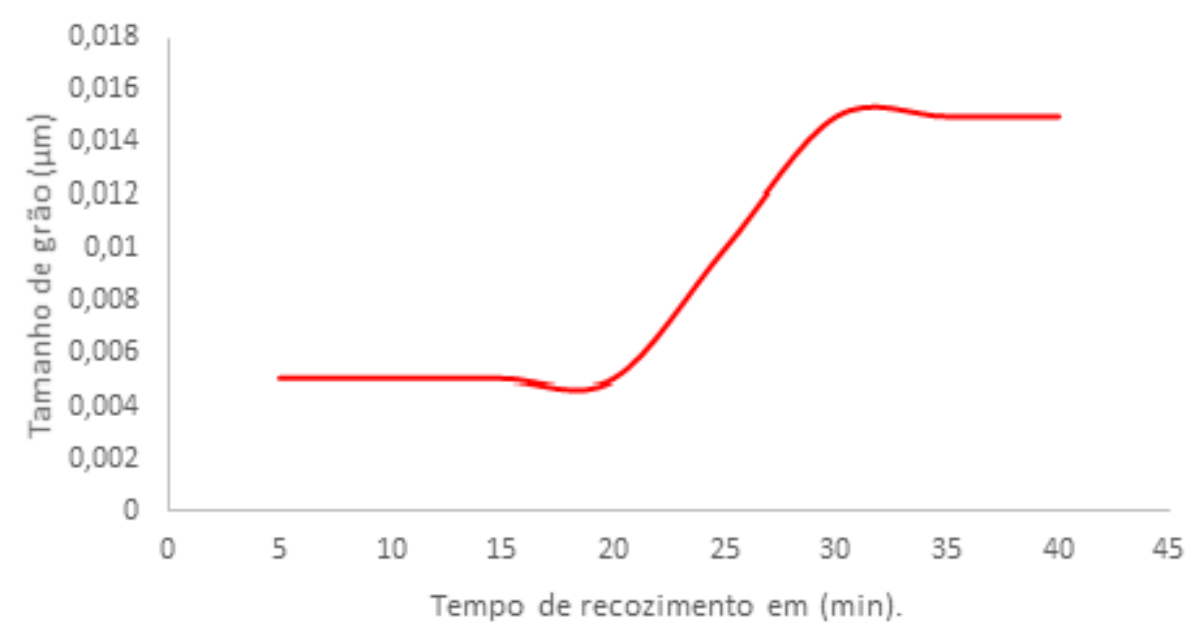

Figura 2. Evolução do tamanho de grão durante o recozimento a $450^{\circ} \mathrm{C}$.

Comparando a figura 2, com a curva de amolecimento é possível verificar que o mesmo ocorre um aumento gradativo no tamanho do grão a dureza do latão varia um pouco.

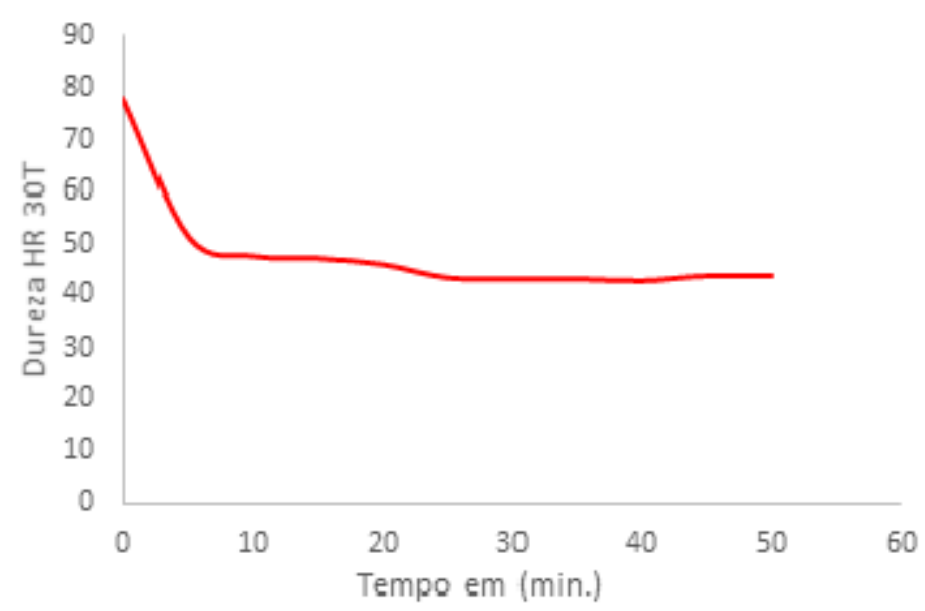

Figura 3. Curva de amolecimento a $450^{\circ} \mathrm{C}$.

A Fração recristalizada foi mensurada ao longo de cada tempo estudado e calculados os valores de $\ln \left(-\ln \left(1-V_{v}\right)\right.$ e $\ln (t)$. Após a plotagem dos pontos, efetuou-se uma regressão linear para obter a reta que melhor se ajustava aos resultados. Traçado a reta foi possível a obtenção dos valores de $n$ e $k$ da equação de JMAK :1,52 e 2,52 
x $10^{-4}$ esses valores indicam uma taxa de nucleação e crescimento dos grãos constantes, portanto e possível concluir que o modelo JMAK satisfaz esta experiencia.

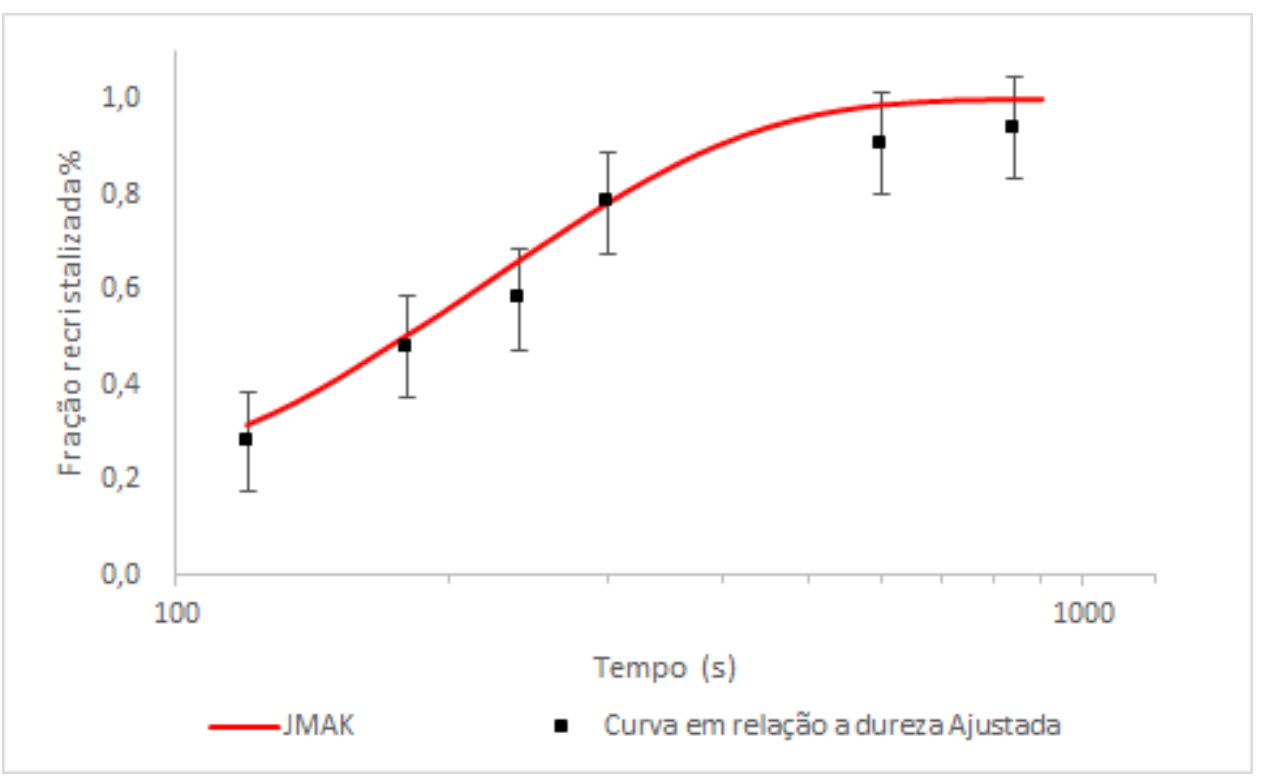

Figura 4. Curva da fração recristalizada em função do tempo de recozimento para a temperatura de $450^{\circ} \mathrm{C}$.

Winning e Schafer [2] estudaram a influência da recristalização nas propriedades mecânicas, utilizando a formula que correlaciona a fração recristalizada com a dureza formula descrita na equação 2 .

$\underline{H v(0)-H v(t)}$

$\mathrm{Hv}_{(0)}-\mathrm{Hv}($ rec. $)$

Onde $\mathrm{Hv}_{(0)}$ e a dureza na condição inicial, $\mathrm{Hv}_{(t)}$ e a dureza nos diferentes tempos e $\mathrm{Hv}_{(\text {rec })}$ e a dureza após a recristalização estar completa considerou-se a dureza após 25 minutos de tratamento térmico. A recristalização é uma fase que ocorre a nucleação e 0 crescimento, portanto sua cinética e semelhante à das outras transformações heterogêneas ${ }^{[3]}$. A cinética de recristalização se aproximou do comportamento sigmoidal obedecendo a equação JMAK onde as taxas de crescimento foram aproximadamente constantes ${ }^{[4]}$. O resultado sigmoidal pode ser dividido em três partes ${ }^{[3]}$.O material começou a recristalizar lentamente (período de incubação), depois aumentou sua velocidade até um valor de máxima nucleação (período de máxima aceleração) e finalmente, voltou a ser lenta (período de desaceleração).Neste trabalho o acompanhamento da evolução microestrutural foi utilizado o ensaio de dureza submetidos a diferentes condições de tratamento térmico. Os resultados obtidos nesse experimento em relação a temperatura, que as transformações microestruturais evoluem mais lentamente em temperaturas menores, que justifica pela alta energia de ativação para recristalização neste domínio(redução significativa da difusão em função da temperatura), está de acordo com os estudos feitos por Gorelik ${ }^{[6]}$ e Lima ${ }^{[7]}$ feitos com as ligas do Al-Si e Al-Fe-Si, respectivamente. 


\section{CONCLUSÃO}

A cinética de recristalização para a liga C272000 estudada foi descrita utilizando as equações das frações recristalizadas obtidas através das variações das propriedades mecânicas que apresentaram boa correlação com o modelo matemático JMAK a recristalização de $78 \%$ em 5 minutos e totalmente recristalizada a 20 minutos e a correlação com o experimento mecânico feito por dureza a recristalização de $78 \%$ em 5 minutos e totalmente recristalizada a 15 minutos, logo o modelo matemático JMAK satisfaz o experimento.

\section{Agradecimentos}

Os autores agradecem a empresa PARANAPAMENA por fornecer o material, o IFSP por apoiar este trabalho, minha esposa e filhos por incentivar a estudar e a Deus por proporcionar a oportunidade de participamos deste estudo.

\section{REFERÊNCIAS}

[1] MC da Silva Filho., JFC Lins., PR Rios., Estudo da cinética de recristalização de um aço microligado processada via ARB - ResearchGate - 2009.

[2] WINNING, M.; SCHAFER, C. Influencing Recrystallization Beauvoir by mechanical loads. Materials Science and EngineeringA, v. 419, n. 1-2, p. 18-24, Mar. 2006.

[3] PB Guimarães, YP Yadava, RAS Ferreira., Influência do tempo de recozimento sobre a morfologia da fratura e recristalização na liga de alumínio AA 8023 - Revista Eletrônica de $\ldots, 2011$

[4] VANDERMEER, R.; MASURA, R.A.; RATH, B.B. Microstructural paths of shapepreserved nucleation and growth transformations. Acta Metallurgica et Materialia, v. 39, n. 3, p. 383-9, 1991.

[5] TSUJI, N.; UEJI, R.; MINAMINO, Y. Nanoscale crystallographic analysis of ultrafine grained IF steel fabricated by ARBprocess. Scripta Materialia, v. 47, n. 2, p. 69-76, 2002.

[6] Gorelik, S. S., Recrystallization in Metals and Alloys. MIR

Publishers, Moscow, p. 7-117; 444-464, 1981.

[7] Lima, E. P. R., Sanguinetti Ferreira, R. A., Quadros, N.F., Yadava, Y. P., Estudo dos Aspectos Cinéticos e Morfológicos Durante Recristalização da Liga de Alumínio AA 8011, Revista Iberoamericana de Engeniería Mecânica, vol. 10, n¹, p.131-137, 2006.

[8] Guimarães, P. B., Assunção, T. L., Yadava, Y. P.,Ferreira, R. A. S., A Cinética de Recristalização da Liga de Alumínio AA 8023 Recozida em Diferentes Temperaturas. In: 18 CBECiMat - Congresso Brasileiro de Ciência e Engenharia de Materiais, Porto de GalinhasPE, p. 8917-89252008.

[9] SILVA FILHO, M.C.; LINS, J.C.F.; RIOS, P.R.; BOTT, I.S. Caracterização microestrutural de um aço micro ligado estabilizado ao nióbio processado via ARB e posteriormente recozido a $600^{\circ} \mathrm{C}$. In: CONGRESSO ANUAL DA ABM, 63.,2008, Santos, SP. Anais... São Paulo: ABM, 2008. p. 3038-47. 